\title{
LOW-POWER HIGH-ORDER BAND-PASS ACTIVE-RC ALLPOLE FILTERS USING A "LOSSY" LP-BP TRANSFORMATION
}

\author{
Dražen Jurišić $^{1}$, Neven Mijat ${ }^{1}$ and George S. Moschytz ${ }^{2}$
}

${ }^{1}$ University of Zagreb, Department of Electronic Systems and Information Processing, Unska 3, HR-10000 Zagreb, Croatia. E-mail: drazen.jurisic@,FER.hr, neven.mijat@,FER.hr

${ }^{2}$ Swiss Federal Institute of Technology Zürich (ETH), Signal and Information Processing Laboratory (ISI), Sternwartstrasse 7, CH-8092 Zürich, Switzerland. E-mail: moschytz@isi.ee.ethz.ch

\begin{abstract}
The design procedure for high-order single amplifier BP filters is presented. A method for the design of $2^{\text {nd }}$ - and $4^{\text {th }}$-order band-pass (BP) active$R C$ filters using a modified low-pass to band-pass (LP$\mathrm{BP})$ frequency transformation, was already presented in previous works [1],[2]. It was shown that a BP filter could be realized simply by the substitution of resistors and capacitors of the ladder in a low-pass (LP) prototype filter, by serial and parallel $R C$ circuits. Such a substitution results from a so-called, "lossy" LP-BP transformation. In this paper, the design procedure is extended to higher-order BP filters, such as $6^{\text {th }}$ - and $8^{\text {th }}$ order. The design procedure is simple, and the closedform design equations, are presented. Furthermore, it is shown that "impedance tapering" decreases sensitivities to component tolerances for the LP prototype, as well as for the resulting BP filter. The Schoeffler's sensitivity measure is used for the sensitivity analysis.
\end{abstract}

Index Terms: Allpole filters, BP filters, LP-BP transformation, low-sensitivity active filters

\section{INTRODUCTION}

The design of BP filters is usually performed by means of the well-known LP-BP frequency transformation applied to a LP prototype filter transfer function. The advantage of a passive- $L C$ filter realization lies in the existence of the corresponding reactance LP-BP transformation, which defines the $\mathrm{BP}$ filter structure enabling a straightforward realization procedure, and the calculation of the element values. In previous works, it was shown that it is possible to find impedance LP-BP transformation by an active- $R C$ filter, too. The design procedure is already derived for the $2^{\text {nd }}$ - and $4^{\text {th }}$-order BP filters, which were obtained applying the so-called "lossy" LP-BP transformation as presented in [1], [2]. The circuits are of the class-4, i.e. they have an $R C$-ladder network in the positive feedback loop [3], [4]. In this paper we extend the procedure to the realization of an active- $R C \mathrm{BP}$ filter of higher-order, i.e. $6^{\text {th }}$ - and $8^{\text {th }}$-order directly from a given $3^{\text {rd }}$ - and $4^{\text {th }}$-order LP prototype, using the same impedance transformation. It turns out, however, that there are some differences between the procedures applied to odd-order LP prototype circuits and even order ones. They follow from the fact that the oddorder LP prototype has a real pole and one or more pairs of complex-conjugate poles, while even-order prototype has only complex pole pairs. As a consequence, the realization of a BP filter, from an even-order LP prototype is more straightforward. Both procedures are demonstrated by the realization of $6^{\text {th }}$ and $8^{\text {th }}$-order BP single amplifier filters.

It is shown in [5] that the "impedance tapering" design technique produces low-sensitivity allpole filters, used as LP prototypes. Furthermore, the application of the "lossy" LP-BP transformation to an impedance tapered LP filter, results in a low sensitivity BP filter as well (see [6]). In other words sensitivity to component tolerances of the BP filter is decreased in the same way as the sensitivity of the original LP prototype.

The improvement comes with no additional cost, i.e. it requires only the selection of appropriate component values. This is verified by the use of the Schoeffler's sensitivity measure.

\section{LP-BP TRANSFORMATION}

An even $n^{\text {th }}$-order LP prototype transfer function can be written in the form

$$
T_{L P}(s)=\prod_{i=1}^{n / 2} \frac{K_{i} \cdot \omega_{p i}^{2}}{s^{2}+\left(\omega_{p i} / q_{p i}\right) s+\omega_{p i}^{2}},
$$

while an odd $n^{\text {th }}$-order transfer function has the form

$$
T_{L P}(s)=\frac{K_{\gamma} \cdot \gamma}{s+\gamma} \cdot \prod_{i=1}^{(n-1) / 2} \frac{K_{i} \cdot \omega_{p i}^{2}}{s^{2}+\left(\omega_{p i} / q_{p i}\right) s+\omega_{p i}^{2}},
$$

where $\omega_{p i}$ are pole frequencies and $q_{p i}$ pole Q-factors, and $\gamma$ is a frequency of the negative real pole. The LPBP frequency transformation defined by

$$
s \rightarrow \frac{s^{2}+\omega_{0}^{2}}{B s},
$$

gives a BP filter with the center frequency $\omega_{0}$ and the bandwidth $B$. The application of (3) to (1) and (2), doubles the filter's order obtaining a symmetrical $2 n^{\text {th }}$ order BP filter with the transfer function

$$
T_{B P}(s)=\frac{K \cdot b_{n} s^{n}}{s^{2 n}+a_{2 n-1} s^{2 n-1}+\ldots+a_{1} s+a_{0}} .
$$

The transfer function (4) can be represented as a product of $n$ biquadratic transfer functions of the form:

$$
T_{B P}(s)=\prod_{i=1}^{n} \frac{K_{i}\left(\omega_{i} / q_{i}\right) \cdot s}{s^{2}+\left(\omega_{i} / q_{i}\right) \cdot s+\omega_{i}^{2}} .
$$

For odd $n$, there is a factor whose pole-Q, $q_{1}$, is given by 


$$
q_{1}=\omega_{0} /(B \gamma),
$$

and the pole frequency $\omega_{1}$ is given by

$$
\omega_{1}=\omega_{0} \text {. }
$$

The rest of the product (5) contains the factor pairs having equal Q-factors $q_{i 1}=q_{i 2}=q_{i}$ defined by

$$
q_{i}=\sqrt{\frac{\left(4+B^{2} \omega_{p i}^{2} / \omega_{0}^{2}\right)+\sqrt{\left(4+B^{2} \omega_{p i}^{2} / \omega_{0}^{2}\right)^{2}-4\left(B^{2} \omega_{p i}^{2}\right) /\left(q_{p i}^{2} \omega_{0}^{2}\right)}}{2\left(B^{2} \omega_{p i}^{2}\right) /\left(q_{p i}^{2} \omega_{0}^{2}\right)}}
$$

and pole frequency pairs $\omega_{i 1}, \omega_{i 2}$, which are given by

$$
\frac{\omega_{i 1}}{\omega_{0}}=\frac{\omega_{0}}{\omega_{i 2}}=\frac{B q_{i} \omega_{p i} / \omega_{0}+\sqrt{\left(B q_{i} \omega_{p i} / \omega_{0}\right)^{2}-4 q_{p i}^{2}}}{2 q_{p i}}
$$

A procedure for the realization of an active- $R C \mathrm{BP}$ filter, which uses the "lossy" LP-BP transformation, pre-warps the pole frequencies and pole-Q factors of a given normalised LP prototype, and uses the prototype impedance transformation presented in Fig. 2 [1], [2]. The most important steps and design formulas will be repeated here and used in the design of higher-order, i.e. $6^{\text {th }}$ - and $8^{\text {th }}$-order BP filters.
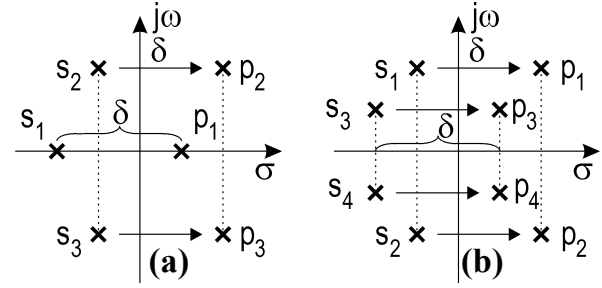

Fig. 1 The transformation of s-variable, i.e. LP filter prototype pole shift for $\delta$ (a) $3^{\text {rd }}$-order. (b) $4^{\text {th }}$-order.

By introducing a new variable $p=S+\delta$, where $\delta$ is a real positive constant, we apply the transformation

$$
s=p-\delta
$$

to (3) and obtain a new transfer function $T_{1}(p)$, whose poles are shifted to the right by an amount $\delta$ as shown in Fig. 1. The new even $n^{\text {th }}$-order LP filter prototype transfer function is

$$
T_{1}(p)=\prod_{i=1}^{n / 2} \frac{K \cdot \omega_{p i}^{2} \cdot s}{p^{2}+\left(\Omega_{p i} / Q_{p i}\right) p+\Omega_{p i}^{2}},
$$

and odd $n^{\text {th }}$-order

$$
T_{1}(p)=\frac{K_{\gamma} \cdot \gamma}{p+\Gamma} \cdot \prod_{i=1}^{(n-1) / 2} \frac{K_{i} \cdot \omega_{p i}^{2}}{p^{2}+\left(\Omega_{p i} / Q_{p i}\right) p+\Omega_{p i}^{2}},
$$

where

$$
\Gamma=\gamma-\delta, \Omega_{p i}=\sqrt{\omega_{p i}^{2}-\frac{\omega_{p i}}{q_{p i}} \delta+\delta^{2}}, Q_{p i}=\frac{\Omega_{p i}}{\omega_{p i} / q_{p i}-2 \delta} .
$$

Application of $(10)$ to $(3)$, results
transformation in the variable $p$, given by

$$
p \rightarrow \frac{s^{2}+\omega_{0}^{2}}{B s}+\delta \text {. }
$$

It is shown in [1], [2] that the application of the "lossy" LP-BP transformation, transforms resistors in the ladder network of the LP circuit into series $R C$ circuits, and capacitors into parallel $R C$ circuits. This substitution, together with impedance tapering factors $r$ and $\rho$, which are defined in [6],[5], are shown in Fig. 2.

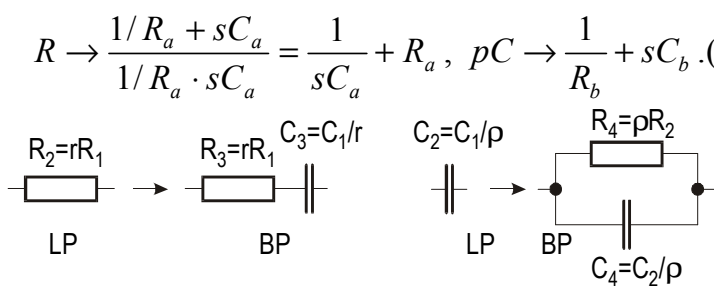

Fig. 2 Impedance tapering factors $r$ and $\rho$ by LP-BP transformation.

The substitution in Fig. 2 replaces the expression $p \cdot R C$ in the transfer function coefficients by:

$$
p \cdot R C \rightarrow \frac{\left(1 / R_{a}+s C_{a}\right)}{1 / R_{a} \cdot s C_{a}}\left(1 / R_{b}+s C_{b}\right) .
$$

Dividing both sides by $R C$ we obtain a "lossy" LP-BP transformation

$$
p \rightarrow \frac{s^{2}+1 /\left(R_{a}^{\prime} C_{a}^{\prime} R_{b}^{\prime} C_{b}^{\prime}\right)}{s \cdot 1 /\left(R_{a}^{\prime} C_{b}^{\prime}\right)}+\frac{R_{a}^{\prime}}{R_{b}^{\prime}}+\frac{C_{b}^{\prime}}{C_{a}^{\prime}} .
$$

Comparing with (12), we obtain

$\omega_{0}^{2}=\frac{1}{R_{a}^{\prime} C_{a}^{\prime} R_{b}^{\prime} C_{b}^{\prime}{ }_{b}}, B=\frac{1}{R_{a}^{\prime} C_{b}^{\prime}{ }_{b}}, \delta=\frac{R_{a}^{\prime}}{R_{b}^{\prime}}+\frac{C_{b}^{\prime}}{C^{\prime}{ }_{a}}$,

where

$$
R^{\prime}{ }_{a}=R_{a} / R ; C^{\prime}{ }_{a}=R \cdot C_{a} ; R^{\prime}{ }_{b}=R_{b}^{\prime} \cdot C ; C^{\prime}{ }_{b}=C_{b} / C
$$

The constant $\delta$ is not entirely free. It was shown in [1], [2], that the sensitivity of the filter amplitude response to component tolerances is minimal for

$$
\delta=\delta_{\min }=2 \omega_{0} / B
$$

For this case we have:

$$
\frac{C_{b}^{\prime}}{C^{\prime}{ }_{a}}=\frac{R_{a}^{\prime}}{R_{b}^{\prime}}=\frac{\delta_{\min }}{2}=\frac{\omega_{0}}{B} .
$$

If, for example, $C^{\prime}{ }_{a}$ is arbitrarily chosen, the rest of the parameters can be easily calculated using

$$
C_{b}^{\prime}=C_{a}^{\prime} \cdot \frac{\omega_{0}}{B}, R_{a}^{\prime}=\frac{1}{\omega_{0} \cdot C_{a}^{\prime}}, R_{b}^{\prime}=\frac{B}{\omega_{0}^{2} \cdot C^{\prime}{ }_{a}} .
$$

\section{THE $6^{\text {th }}$-ORDER BP FILTER}

To construct a $6^{\text {th }}$-order BP filter, a modified $3^{\text {rd }}$-order LP filter shown in Fig. 3 needs to be considered. The filter is also a class- 4 circuit.

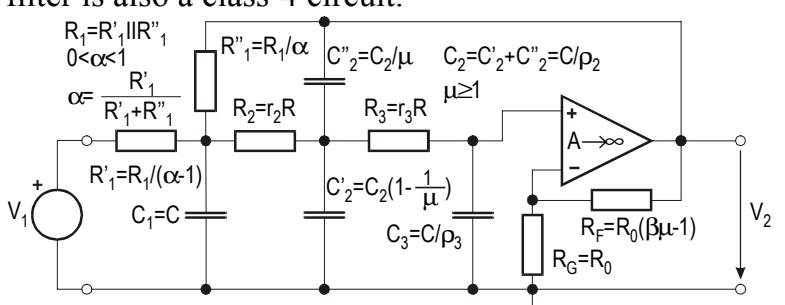

Fig. $33^{\text {rd }}$-order LP filter modified for “lossy" LP-BP transformation.

In comparison to the standard $3^{\text {rd }}$-order LP filter in [5], [4], it is modified by introducing an additional resistor $R_{1}{ }^{\prime}$, which enables shifting of the real pole [1], and additional (optional) capacitor $C_{2}$, , which ensures that the feedback gain $\beta \mu$, where $\mu \geq 1$, is large enough. The capacitor $C_{2}$ ' needs to be added for selective, i.e. narrow-band BP filters (because of theirs high pole-Q factors), while for wide-band filters, with lower pole-Q 
factors, we have $C_{2}{ }^{\prime}=C_{2}$. The voltage transfer function $T(s)$ for the LP prototype circuit has the form given by (2). The coefficients $a_{i}(i=1,2)$ in terms of circuit components are the same as the coefficients of standard $3^{\text {rd }}$-order LP filters [5], [4]. This fact is helpful, because for the design of a filter in Fig. 3, the same equations [5] used for designing a standard $3^{\text {rd }}$-order LP filter can be used here as well. The only difference is in the coefficient $a_{0}$, which is

$$
a_{0}{ }^{\prime}=\frac{1-\alpha \beta \mu}{R_{1} R_{2} R_{3} C_{1} C_{2} C_{3}} .
$$

The coefficient $a_{0}$ ' defined by (21) has the numerator, which enables a shift of the real pole to the right. Using $\Omega_{p}, Q_{p}$ and $\Gamma$, coefficients of the new $T_{1}(p)$ are

$$
a_{0}^{\prime}=\Omega_{p}^{2} \Gamma ; \quad a_{1}^{\prime}=\Omega_{p}^{2}+\Omega_{p} \Gamma / Q_{p} ; \quad a_{2}^{\prime}=\Gamma+\Omega_{p} / Q_{p} .
$$

We can rewrite the coefficient $a_{0}$ ' in the form:

$$
a_{0}^{\prime}=\Omega_{p}^{2} \Gamma=\Omega_{p}^{2}(\gamma-\delta)=\Omega_{p}^{2} \gamma-\Omega_{p}^{2} \delta=a^{\prime \prime}{ }_{0}-a^{\prime \prime}{ }_{0} \alpha \beta \mu
$$

After some calculation we obtain:

$$
\alpha=\delta /(\gamma \beta \mu), a_{0}{ }^{\prime}=\Omega_{p}{ }^{2} \gamma .
$$

Elements of the filter in Fig. 3 can be calculated using standard design procedures for the $3^{\text {rd }}$-order LP filter, which is given in [5], from "shifted" LP coefficients: $a_{0}$ " given in (24), and $a^{\prime}{ }_{i}(i=1,2)$ given by $(22)$. Note, that we use $a_{0}$,', instead of $a_{0}$ '.

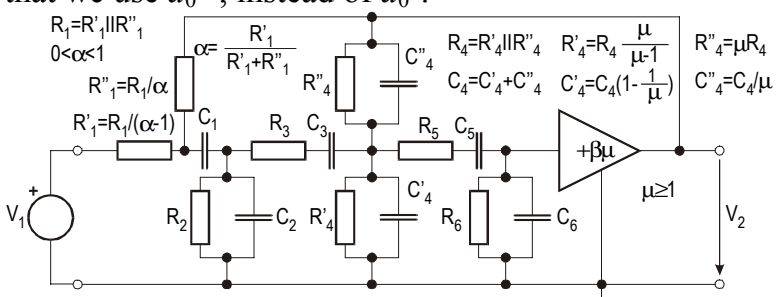

Fig. 4 The $6^{\text {th }}$-order BP filter circuit (using LP-BP transformation).

Example 1) As an illustration the design procedure of the single-amplifier $6^{\text {th }}$-order active- $R C$ BP filter shown in Fig. 4 is considered. The filter is derived from the $3^{\text {rd }}$-order Chebyshev LP filter with normalized cut-off frequency $\omega_{-3 \mathrm{~dB}}=1$ and $0.5[\mathrm{~dB}]$ pass-band ripple, having normalized pole frequencies $\omega_{p}=0.915518$ $[\mathrm{rad} / \mathrm{s}], \gamma=0.536586[\mathrm{rad} / \mathrm{s}]$ and the pole Q-factor $q_{\mathrm{p}}=1.706189$. The BP center frequency is $\omega_{0}=1[\mathrm{rad} / \mathrm{s}]$, and the bandwidth $B=1[\mathrm{rad} / \mathrm{s}]$, or normalized, $B_{n}=B / \omega_{0}=1$. The step-by-step design procedure follows:

i) For given filter specifications, find the $3^{\text {rd }}$-order LP prototype filter pole parameters $\gamma, \omega_{p}$, and $q_{p}$.

ii) Calculate $\delta$ : We choose the minimum value for $\delta$ from (18), i.e. $\delta=\delta_{\min }=2 \omega_{0} / B=2$.

iii) Calculate the new LP prototype by shifting poles by $\delta$. From (11c), the new pole parameters are: $\Omega_{p}=1.94036$ and $Q_{p}=-0.560245$ and $\Gamma=-1.46341$. Note that $Q_{p}$ and $\Gamma$ are negative, i.e., poles lie in the righthalf $p$-plane.

iv) Realize the new LP prototype circuit components:

From (24), calculate $a_{0}$ '" $=2.02025$, and from (22) $a_{1}{ }_{1}=8.83341$ and $a_{2}=-4.92683$. Applying the standard design procedure for the $3^{\text {rd }}$-order LP filter on the circuit in Fig. 3, we choose: $\rho_{2}=3, \rho_{3}=9, C_{1}=C=2, R_{1}=R$ and the LP-prototype filter design frequency (see [5]) $\omega_{d}=(R C)^{-1}=0.15$. Note that $\omega_{d}<\omega_{d \max }=0.204429$ from [5]. We obtain $r_{2}=0.284177, r_{3}=0.158724, \beta=2.17847$ and the normalized value $R_{1}=R=1 /\left(\omega_{d} C\right)=3.333$. After that, we calculate $\alpha$ from (24) to realize $R_{1}$ ' and $R_{1}$ '" and the coefficient $a_{0}$ '. If $\alpha>1$, then we introduce a factor $\mu>1$. From (24) $\alpha=1.71095$, if $\mu=1$. We must, therefore, choose $\mu>1$, for example $\mu=2$, and then we obtain $\alpha=0.855476$, which is realizable by splitting input resistor $R_{1}$ as shown in Fig. 3. With the additional capacitor $C_{2}$ ' we realize $\mu=2$ as presented in Fig. 3. It can be shown that higher values of $\mu$ increase the sensitivities, and $\mu$ should be kept as low as possible.

v) Calculate the transformed impedance component: Let $C^{\prime}{ }_{a}=1$, from (19) we have $C^{\prime}{ }_{b} / C^{\prime}{ }_{a}=\delta_{\min } / 2=1$, and then from (20) $R_{a}{ }_{a}=1 ; C^{\prime}{ }_{b}=1$; and $R^{\prime}{ }_{b}=1$.

vi) Calculate the components of the BP filter: Using normalized $R$ and $C$ values from step $i v$ ), and (17) element values are: $R_{a}=R \cdot R^{\prime}{ }_{a}=3.333 ; C_{a}=C^{\prime}{ }_{a} / R=0.3$; $R_{b}=R^{\prime}{ }_{b} / C=0.5$; and $C_{b}=C \cdot C^{\prime}{ }_{b}=2$. BP filter components are $R_{1}=R_{a} ; R_{2}=R_{b} ; R_{3}=r_{2} R_{a} ; R_{4}=\rho_{2} R_{b} ; R_{5}=r_{3} R_{a} ; R_{6}=\rho_{3} R_{b}$; $C_{1}=C_{a} ; \quad C_{2}=C_{b} ; \quad C_{3}=C_{a} / r_{2} ; \quad C_{4}=C_{b} / \rho_{2} ; \quad C_{5}=C_{a} / r_{3} ;$ $C_{6}=C_{b} / \rho_{3}$. Fig. 5 shows the transfer function magnitude $\alpha(\omega)=20 \log \left|T_{B P}(j \omega)\right|[\mathrm{dB}]$ of the circuit in Fig. 4.

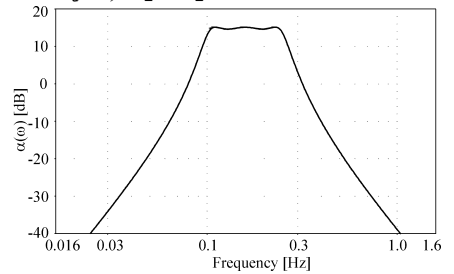

Fig. 5 The $6^{\text {th }}$-order Chebyshev BP filter magnitude. 3.1. Sensitivity analysis of the $6^{\text {th }}$-order BP filter In what follows we show how the impedance-tapering influences sensitivities of the $6^{\text {th }}$-order BP filter. Furthermore, we compare sensitivities of the new highorder single amplifier (1-OA) BP filter to the cascade structure $2^{\text {nd }}$-order BP filter "biquads" (CAS). In order to minimize sensitivities, we apply impedance tapering, to both structures.

Example 2) Consider a BP filter, with normalized central frequency $\omega_{0}=1$, and band-width $B=1[\mathrm{rad} / \mathrm{s}$ ], derived from the $3^{\text {rd }}$-order Butterworth LP prototype. First we apply impedance tapering of capacitors to the LP prototype. The resulting BP filter whose normalized component values are given in Table 1, has a significant improvement of sensitivities.

\begin{tabular}{|c|c|c|c|c|c|c|c|c|c|c|c|}
\hline$\rho_{C}$ & $\omega_{d}$ & $R_{1}$ & $C_{1}$ & $R_{2}$ & $C_{2}$ & $\rho_{2}$ & $\rho_{3}$ & $r_{2}$ & $r_{3}$ & $\alpha$ & $\beta$ \\
\hline 1 & 0.211 & 2.37 & 0.42 & 0.5 & 2 & 1 & 1 & 0.191 & 0.016 & 0.8 & 2.5 \\
\hline 3 & 0.253 & 1.98 & 0.51 & 0.5 & 2 & 3 & 9 & 0.379 & 0.384 & 0.8 & 2.5 \\
\hline 5 & 0.30 & 1.67 & 0.60 & 0.5 & 2 & 5 & 25 & 0.769 & 1.464 & 0.8 & 2.5 \\
\hline
\end{tabular}

Table 1 Normalized component values of BP filters applying capacitive tapering on LP prototype.

The alternative procedure, i.e. tapering the resistors of the LP prototype, is efficient as well. To show this, we consider the BP filter component values in Table 2. For four values of the design frequency $\omega_{d}$, used to 
construct the LP capacitively tapered filter prototype, we obtain the filter with component values in Table 3 .

\begin{tabular}{|c|c|c|c|c|c|c|c|c|c|c|c|}
\hline$r_{R}$ & $\omega_{d}$ & $R_{1}$ & $C_{1}$ & $R_{2}$ & $C_{2}$ & $\rho_{2}$ & $\rho_{3}$ & $r_{2}$ & $r_{3}$ & $\alpha$ & $\beta$ \\
\hline 1 & 0.308 & 1.62 & 0.62 & 0.5 & 2 & 9.197 & 11.11 & 1 & 1 & 0.5 & 4.0 \\
\hline 3 & 0.354 & 1.41 & 0.71 & 0.5 & 2 & 71.69 & 3.00 & 3 & 9 & 0.5 & 4.0 \\
\hline 5 & 0.367 & 1.36 & 0.73 & 0.5 & 2 & 183.5 & 5.00 & 5 & 25 & 0.5 & 4.0 \\
\hline
\end{tabular}

Table 2 Normalized component values of BP filters applying resistive tapering.

\begin{tabular}{|c|c|c|c|c|c|c|c|c|c|c|c|}
\hline No. & $\omega_{d}$ & $R_{1}$ & $C_{1}$ & $R_{2}$ & $C_{2}$ & $\rho_{2}$ & $\rho_{3}$ & $r_{2}$ & $r_{3}$ & $\alpha$ & $\beta$ \\
\hline 1$)$ & 0.225 & 2.22 & 0.45 & 0.5 & 2 & 3 & 9 & 0.273 & 0.375 & 0.73 & 2.73 \\
\hline 2$)$ & 0.254 & 1.97 & 0.51 & 0.5 & 2 & 3 & 9 & 0.384 & 0.384 & 0.80 & 2.49 \\
\hline 3$)$ & 0.275 & 1.82 & 0.55 & 0.5 & 2 & 3 & 9 & 0.498 & 0.376 & 0.86 & 2.32 \\
\hline 4$)$ & 0.30 & 1.67 & 0.60 & 0.5 & 2 & 3 & 9 & 0.699 & 0.348 & 0.95 & 2.11 \\
\hline
\end{tabular}

Table 3 Normalized component values of $B P$ filters applying capacitive tapering with various $\omega_{\text {d. }}$

The alternate procedure, i.e. tapering resistors of the LP prototype results in components given in Table 4.

\begin{tabular}{|c|c|c|c|c|c|c|c|c|c|c|c|}
\hline No. & $\omega_{d}$ & $R_{1}$ & $C_{1}$ & $R_{2}$ & $C_{2}$ & $\rho_{2}$ & $\rho_{3}$ & $r_{2}$ & $r_{3}$ & $\alpha$ & $\beta$ \\
\hline 1$)$ & 0.335 & 1.49 & 0.67 & 0.5 & 2 & 77.7 & 27.7 & 3 & 9 & 0.12 & 16.5 \\
\hline 2$)$ & 0.340 & 1.47 & 0.68 & 0.5 & 2 & 62.6 & 32.9 & 3 & 9 & 0.16 & 12.2 \\
\hline 3$)$ & 0.345 & 1.45 & 0.69 & 0.5 & 2 & 48.7 & 40.5 & 3 & 9 & 0.23 & 8.68 \\
\hline 4$)$ & 0.350 & 1.43 & 0.70 & 0.5 & 2 & 35.8 & 52.8 & 3 & 9 & 0.34 & 5.88 \\
\hline
\end{tabular}

Table 4 Normalized component values of BP filters applying resistive tapering with various $\omega_{d}$

Sensitivity analysis is performed assuming relative changes of resistors and capacitors to be uncorrelated random variables, with a zero-mean Gaussian distribution and 1\% standard deviation. The standard deviation (which is related to Schoeffler's sensitivities) of the variation of the logarithmic gain $\Delta \alpha=8.68588$ $\Delta\left|T_{B P}(\omega)\right| /\left|T_{B P}(\omega)\right|[\mathrm{dB}]$, with respect to passive elements, is calculated for filter examples from Table 1 to Table 4 and shown in Fig. 6 (T- stands for tapering).

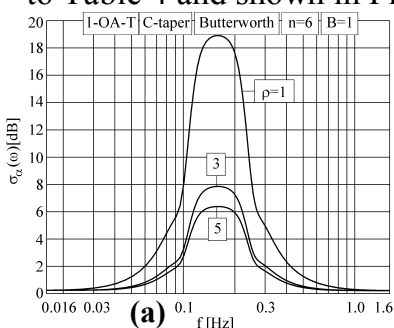

(a) ${ }^{0.1}{ }_{\mathrm{f}[\mathrm{Hz}]}$
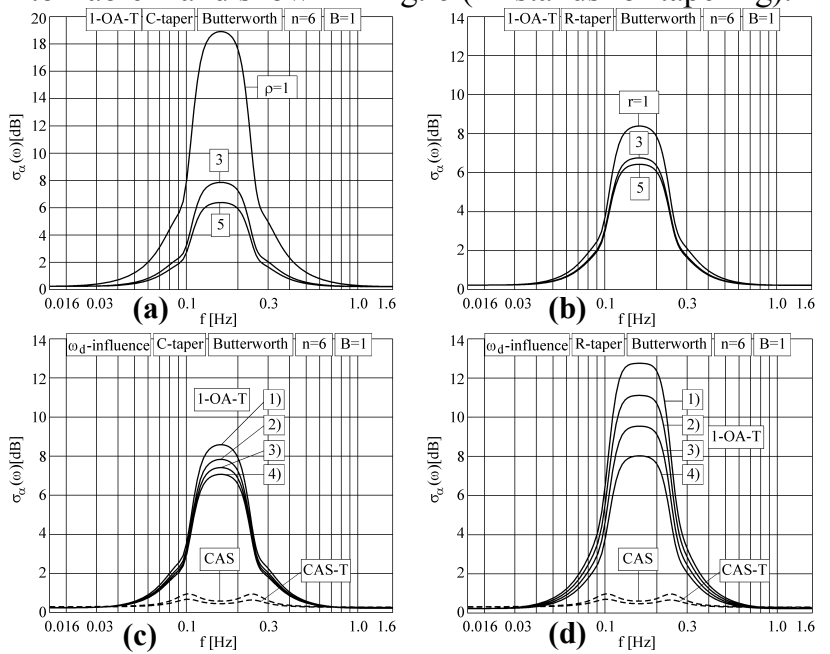

(d) ${ }^{0.1}{ }_{\mathrm{f}[\mathrm{Hz}]}$ filters can be designed applying the impedance tapering and optimising the BP filter, by choosing an optimum value of $\omega_{d}$. For optimum $\omega_{d}$ value there exists min. sensitivity of the BP filter magnitude to component tolerances.
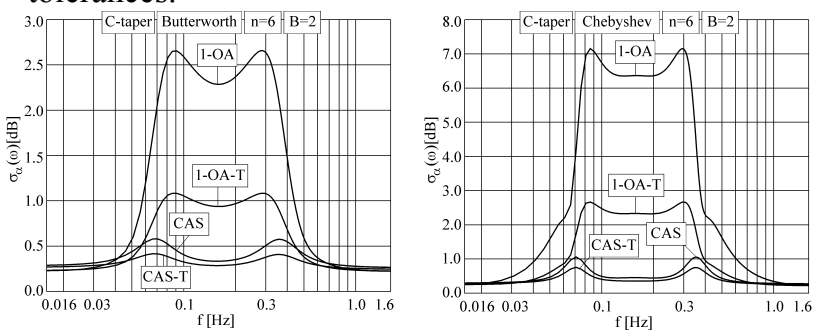

Fig. 7 The sensitivity comparison of the singleamplifier $6^{\text {th }}$-order BP filter circuit to the cascade; (b) Butterworth $B=2$. (d) Chebyshev $B=2$.

In Fig. 7, we present sensitivities of various approximations (Butterworth and Chebyshev) and bandwidth $B=2$ compared to the cascade design. Higher pole-Qs in Chebyshev approximations inevitably produce higher sensitivities than Butterworth. In all cases the cascade design has lower sensitivity. Comparing to the narrow pass-band $B=1$ case in Fig. 6, which has high sensitivities, we can conclude that only impedance tapered 1-OA BP filters with the wider pass-band have low sensitivities, which are near to sensitivities of a cascade design and, therefore, are useful for the 1-OA BP filter realisation. This is even more restrictive in higher-order BP filters, for example $8^{\text {th }}$-order, the design of which will be shown in what follows.

\section{THE $8^{\text {th }}$-ORDER BP FILTER}

In order to realize an $8^{\text {th }}$-order BP filter, consider the $4^{\text {th }}$-order LP filter shown in Fig. 8.

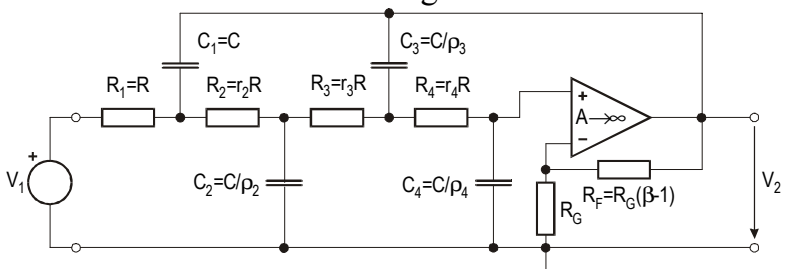

Fig. 8 The $4^{\text {th }}$-order LP prototype filter.

The realization of the $8^{\text {th }}$-order BP filter shown in Fig. 9 , is simpler than the realization of the $6^{\text {th }}$-order BP filter, because a pair of complex-conjugate poles can be shifted into the right by an amount $\delta$, as shown in Fig. 1 (b), simply by increasing the gain value $\beta$. Sometimes, for large values of $\delta$, the pre-warped poles can lie in the right-half plane. ${ }^{\dagger}$ It does not produce stability problems, because the shift $\delta$ is cancelled in next design steps.

Example 3) As an illustration of the proposed BP filter design procedure, a $4^{\text {th }}$-order Chebyshev LP prototype filter with $\omega_{-3 \mathrm{~dB}}=1$ and $0.5[\mathrm{~dB}]$ ripple is considered.

\footnotetext{
† It is realizable, because the root-locus diagram of the class-4 filters, have branches in the right-half plane for enough large values of the gain $\beta$.
} 
The BP filter center frequency is $\omega_{0}=1[\mathrm{rad} / \mathrm{s}]$, and $B=1$ $[\mathrm{rad} / \mathrm{s}]$. The step-by-step design procedure follows:

i) Define the $4^{\text {th }}$-order LP prototype filter pole parameters $\omega_{p 1}, \omega_{p 2}$, and $q_{p 1}, q_{p 2}$. They are: $\omega_{p 1}=0.9434$ $[\mathrm{rad} / \mathrm{s}], \omega_{p 2}=0.5461[\mathrm{rad} / \mathrm{s}], q_{\mathrm{p} 1}=2.9405$ and $q_{\mathrm{p} 2}=0.7051$. ii) Calculate the minimum value of $\delta: \delta=\delta_{\min }=2 \omega_{0} / B=2$.

iii) Calculate the LP prototype by shifting the poles by $\delta$ : Its pole parameters are: $\Omega_{p 1}=2.06116, Q_{p 1}=-0.56023$, $\Omega_{p 2}=1.65806$, and $Q_{p 2}=-0.514057 . Q_{p 1}$ and $Q_{p 2}$ are $<0$, i.e., the poles lie in the right-half $p$-plane.

iv) Realize the new LP prototype circuit components:

From $\Omega_{p i}, Q_{p i}(i=1,2)$ calculate coefficients of new $T_{1}(p)$, i.e. $a_{0}{ }^{\prime}=11.6795, a^{\prime}{ }_{1}=-23.8175, a_{2}{ }_{2}=18.8645$ and $a_{3}=-6.9046$. Applying the standard (numerical) design procedure for the $4^{\mathrm{th}}$-order LP filter and choosing: $\rho_{2}=3, \rho_{3}=9, \rho_{3}=27, C_{1}=C=1$, and $\omega_{d}=0.15$, we obtain $r_{2}=0.2685, r_{3}=1.0088, r_{4}=0.1166, \beta=1.6523$ and the normalized value $R_{1}=R=1 /\left(\omega_{d} C\right)=6.667$.

v) Calculate the transformed impedance component: Let $C^{\prime}{ }_{a}=1$, from (19) we have $C^{\prime}{ }_{b} / C^{\prime}{ }_{a}=\delta_{\min } / 2=1$, and then from (20) $R_{a}{ }_{a}=1 ; C^{\prime}{ }_{b}=1$; and $R^{\prime}{ }_{b}=1$.

vi) Calculate the components of the $8^{\text {th }}$-order BP filter: From the normalized $R$ and $C$ values [step $i v)$ ], and (17) the element values are $R_{a}=R \cdot R^{\prime}{ }_{a}=6.667 ; C_{a}=C^{\prime}{ }_{a} / R=0.15$ $R_{b}=R^{\prime}{ }_{b} / C=1$; and $C_{b}=C \cdot C^{\prime}{ }_{b}=1$. The BP filter components are $R_{1}=R_{a} ; R_{2}=R_{b} ; R_{3}=r_{2} R_{a} ; R_{4}=\rho_{2} R_{b} ; R_{5}=r_{3} R_{a}$; $R_{6}=\rho_{3} R_{b} ; R_{7}=r_{4} R_{a} ; R_{8}=\rho_{4} R_{b} ; C_{1}=C_{a} ; C_{2}=C_{b} ; C_{3}=C_{a} / r_{2} ;$ $C_{4}=C_{b} / \rho_{2} ; C_{5}=C_{a} / r_{3} ; C_{6}=C_{b} / \rho_{3}, C_{7}=C_{a} / r_{4} ; C_{8}=C_{b} / \rho_{4}$.

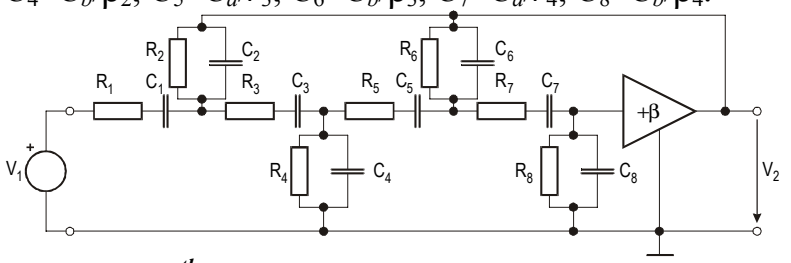

Fig. 9 The $8^{\text {th }}$-order BP filter circuit.

4.1. Sensitivity analysis of the $8^{\text {th }}$-order BP filter Sensitivities of the $8^{\text {th }}$-order BP filter, shown in Fig. 9, with Butterworth and Chebyshev approximations, and band-width $B=2[\mathrm{rad} / \mathrm{s}]$ compared to the cascade design (CAS) are presented in Fig. 10.
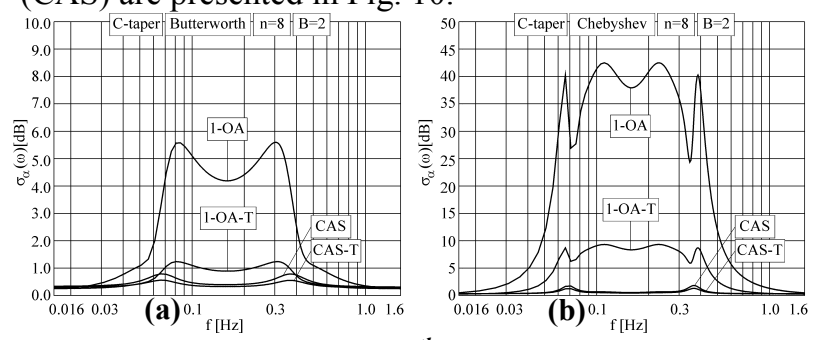

Fig. 10 Sensitivities of the $8^{\text {th }}$-order BP filter circuit with; $\omega_{0}=1, B=2$. (a) Butterworth. (b) Chebyshev.

The narrow pass-band BP filters (for example with $B=1$ ), have higher pole-Q values than wider pass-band $\mathrm{BP}$ filters (with $B=2$ ) resulting in extremely high sensitivities thus they are not presented. From Fig. 10, we conclude that BP filters with $B=2$ (specially those with "low-Q" Butterworth approximation), that are impedance tapered, have acceptably low sensitivities, which are near those realized by a CAS. Consequently,
BP filters with narrow pass-band, and approximations with higher pole-Q factors, are not suitable for the realization using our new 1-OA BP filter topology. This is particularly true for high-order BP filters.

\section{CONCLUSIONS}

A procedure for the design of allpole low-sensitivity, low-power high-order $\left(6^{\text {th }}\right.$ and $\left.8^{\text {th }}\right)$ BP active- $R C$ filters using impedance tapering is presented. The filters are designed using a "lossy" LP-BP transformation [2]. The filters use only one operational amplifier, and a minimum number of passive components. The design procedure is straightforward and the calculation of the BP filter components is very simple. Using the appropriate impedance tapering factors $r_{i}, \rho_{i}$, and the design frequency $\omega_{d}$, while designing LP prototype filter, we can reduce the sensitivity to component tolerances of the resulting BP filter. The reduction in power and component count achieved with the singleamplifier $6^{\text {th }}$ - and $8^{\text {th }}$-order BP filters is obtained at a price: a cascade of impedance-tapered biquads is better on sensitivity performance. Thus the decision on which way to go is typically one of tradeoffs: low power and component count versus low sensitivity. The component count increases, and sensitivities get worse, when we realize selective, narrow-band BP filters with our new 1-OA filter. We conclude that the new $6^{\text {th }}$ - and $8^{\text {th }}$-order filters are most suitable for the realization of (non-selective) wide-band BP filters of low-power and low component count. Otherwise, it is recommended to use other low sensitivity structures for the realization of (selective) narrow-band BP filters, such as: the cascade of impedance-tapered biquads, leap-frog, follow-theleader feedback, cascade of biquarts, etc.

\section{REFERENCES}

[1] D. Jurišić and N. Mijat, "Use of "Lossy" LP-BPTransformation in Active Second-order BP Filter Design Procedure," in Proc. of ISPA 2001, (Pula, Croatia), June 19-21, 2001, pp. 491-495.

[2] D. Jurišić, N. Mijat and G. S. Moschytz, "Design of Fourth-Order Band-Pass Active-RC Filters Using a "Lossy" LP-BP Transformation," in Proc. of ECCTD 2001, (Espoo, Finland), Vol. 1, August 28 31, 2001, pp. 117-120.

[3] R. P. Sallen and E. L. Key, "A practical Method of Designing RC Active Filters," IRE Transactions on Circuit Theory, vol. CT-2, 1955, pp. 78-85.

[4] G. S. Moschytz, Linear Integrated Networks: Design, New York (Bell Laboratories Series): Van Nostrad Reinhold Co., 1975.

[5] G. S. Moschytz, "Low-Sensitivity, Low-Power, Active- $R C$ Allpole Filters Using Impedance Tapering," IEEE Trans. on Circ. and Syst. II, vol. CAS-46, No. 8, Aug. 1999, pp. 1009-1026.

[6] D. Jurišić, G. S. Moschytz and N. Mijat, "LowSensitivity, Low-Power Fourth-Order Band-Pass Active-RC Allpole Filter Using Impedance Tapering," in Proc. of ICECS 2001, (Msida, Malta), Vol. 2, September 2 - 5, 2001, pp. 815-818. 
